\title{
Adaptive Yaw Control of Three-axle Road Vehicles Based on Mass, Yaw Inertia and CG Position Identification
}

\author{
Yubiao Zhang*, Ami Woo, Baris Fidan, Amir Khajepour \\ Department of Mechanical and Mechatronics Engineering \\ University of Waterloo \\ Waterloo, Ontario, Canada
}

\begin{abstract}
This paper introduces an adaptive yaw control scheme based on the estimation of the vehicle mass, yaw inertia and center of gravity (CG) position. The control deigns for three-axle road vehicles, which can be trucks, buses, or even three-axle passenger cars. System parameters of these vehicles vary significantly due to varying conditions, such as unloading and fully-loading of payloads. As a result, control references and fixed-model-based controller lose efficacy. The proposed adaptive yaw control compensates these issues, utilizing the integration of a least-square based parameter identification algorithm and a Model Reference Adaptive Control (MRAC) law. Simulation test results verify the effectiveness of the proposed adaptive control scheme.
\end{abstract}

Keywords-three-axle vehicle, vehicle dynamics, parameters identification, adaptive yaw control

\section{INTRODUCTION}

Advances in low cost sensors and computation technology expedited the progress on the performance and control of modern vehicles. One of the active areas of automobile research so far has been vehicle stability and handling characteristics. A practical and proven solution to improve the vehicle dynamics during critical driving conditions has been to produce corrective yaw moment to the vehicle. Various control methods to direct yaw-moment control (DYC) has been proposed in literature including electronic stability program based on differential braking $[1,2]$ and differential traction or torque vectoring [3, 4]. Indirect yaw moment control systems such as active steering control, which produces corrective steering angle to the wheels, have been alternative way to stabilize yaw moment $[5,6]$.

In vehicle control designs, the vehicle dynamics are often estimated using single track model. To simplify the controller design process, assumptions such as the negligible effect of the vehicle parameter variations on the dynamics and the performance region of the tire forces in the linear range are often imposed. Therefore, the performance of many existing control designs are promising specifically on two axle vehicles travelling within the linear range of the tires. However, when the effect of the vehicle parameter variations on the performance becomes substantial, the controller becomes less effective even under the same operating conditions.
One of the important parameters to take into consideration in control design is the vehicle mass. With varying mass, the center of gravity and inertia of vehicle vary, which has direct effect on the overall vehicle dynamics. For some vehicles such as passenger cars where the variation of the payload compared to the mass of the vehicle is insignificant, such changes may be tolerable. These variations are more critical in situations when the variation of the loading conditions and configurations impacts more on the overall dynamics [7-9]. The three axle heavy load trucks and commercial buses typically involve in multiple loadings and unloading of payloads more significantly compared to two axle passenger cars. These variations affect the stability and performance of the overall vehicle motion even with the appropriate control design. A controller which adapts to such changes and responds accordingly is desired. The apparent difficulty with variation of parameters in the process of control design is that these parameters are not directly measurable.

In this paper, an adaptive DYC is proposed on three-axle vehicles utilizing least-square (LS) estimation of unknown parameters of the vehicle, for improving the trajectory tracking of yaw dynamics. Section II explains the three-axle vehicle system dynamics. A single track model is developed to describe the DYC system. In section III, parameter identification and control design are developed. The mass, inertia, and center of gravity of vehicle are treated as unknown parameters and estimated indirectly in the section. The model reference adaptive control design is proposed with the reference model. The performance and effectiveness of the control strategy is proposed in Section IV. Finally, the paper is summarized and concluded in Section V.

\section{VEHICLE MODELING}

\section{A. Single Track Model}

The vehicle dynamics, in general, is non-linear and is difficult to formulate precisely. For vehicle state estimation and motion control design, linearized models are preferred. A three-axle vehicle single track model $[10,11]$, depicted in Fig. 1 is used in this paper The equations for lateral dynamics and yaw motion are expressed by

$$
\begin{gathered}
m a_{y}=F_{y f}+F_{y c}+F_{y r}, \\
I_{z} \dot{r}=F_{y f} l_{f}-F_{y c} l_{c}-F_{y r} l_{r}+M_{e}+M_{d},
\end{gathered}
$$

*email: gary.zhang@uwaterloo.ca 


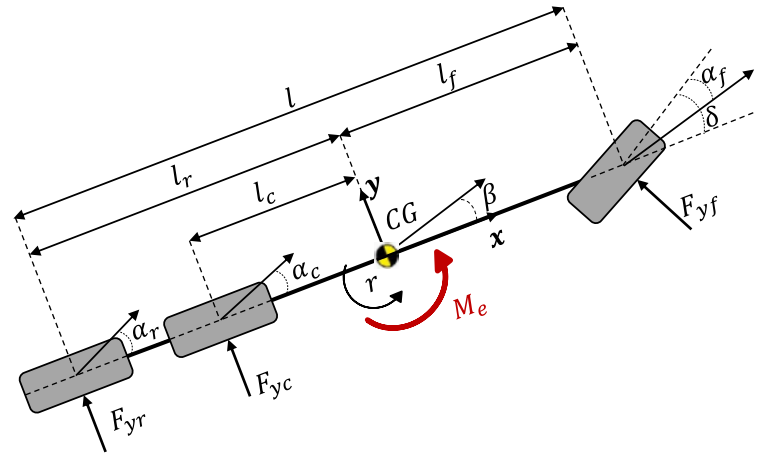

Fig. 1 The single track model

and the lateral acceleration is given by

$$
a_{y}=\dot{v}_{y}+v_{x} r=v_{x}(\dot{\beta}+r),
$$

where, assuming the slip angles are small, tire lateral forces have a linear relationship to the tire slip angle as

$$
\begin{aligned}
& F_{y f}=C_{f}\left(\delta-\beta-\frac{l_{f} r}{v_{x}}\right) \\
& F_{y c}=C_{c}\left(-\beta+\frac{l_{c} r}{v_{x}}\right) \\
& F_{y r}=C_{r}\left(-\beta+\frac{l_{r} r}{v_{x}}\right)
\end{aligned}
$$

The terms in (1)-(6) are defined as follows:

$m, I_{z}$ - the vehicle mass and the moment of inertia about $\mathrm{z}$ axis;

$v_{x}, v_{y}, v$ - vehicle longitudinal speed, lateral speed and vehicle resultant speed;

$\alpha_{y}, \beta, r$ - lateral acceleration, body slip angle and yaw rate at vehicle's CG (center of gravity);

$F_{y f}, F_{y c}, F_{y r}$ - lateral force of front, central and rear tires;

$l_{f} / l_{c} / l_{r} / l$ - the distance from CG to front/central/rear axle, the distance between front axle to rear axle;

$C_{f} / C_{c} / C_{r}$ - the front/central/rear wheels cornering stiffness,

$\delta$ - the front wheels steering angle;

$M_{e}$ - the extra yaw moment from the controller;

$M_{d}$ - the disturbance moment, the side wind causes this disturbance moment and affect to the vehicle's stability in cornering maneuver, It is assumed to be zero in this research.

Rearranging (1)-(6), the yaw dynamics at a certain operating point can be represented by the following state space

$$
\begin{gathered}
\dot{x}=A x+B u \\
y=x
\end{gathered}
$$

where $x=\left[\begin{array}{ll}\beta & r\end{array}\right]^{T}, u=\left[\begin{array}{ll}\delta & M_{e}\end{array}\right]^{T}$, and the output $y$ is as the same as the system state;

$$
A=\left[\begin{array}{cc}
-\frac{C_{f}+C_{c}+C_{r}}{m v_{x}} & -1-\frac{l_{f} C_{f}-l_{c} C_{c}-l_{r} C_{r}}{m v_{x}^{2}} \\
-\frac{l_{f} C_{f}-l_{c} C_{c}-l_{r} C_{r}}{I_{z}} & -\frac{l_{f}^{2} C_{f}+l_{c}^{2} C_{c}+l_{r}^{2} C_{r}}{I_{z} v_{x}}
\end{array}\right], B=\left[\begin{array}{cc}
\frac{C_{f}}{m v_{x}} & 0 \\
\frac{l_{f} C_{f}}{I_{z}} & \frac{1}{I_{z}}
\end{array}\right] .
$$

\section{B. Model Validation}

The derived model (7) was simulated for verification with the high-fidelity model of TruckSim with step-steer input (constant speed of $80 \mathrm{~km} / \mathrm{h}$ ) with bus parameters presented in Table 1. The result in Fig. 2 shows that the vehicle dynamics has a fast response and small overshoot. But they are quite similar with the results of the TruckSim model, which verifies the reliability of the derived model for control design.
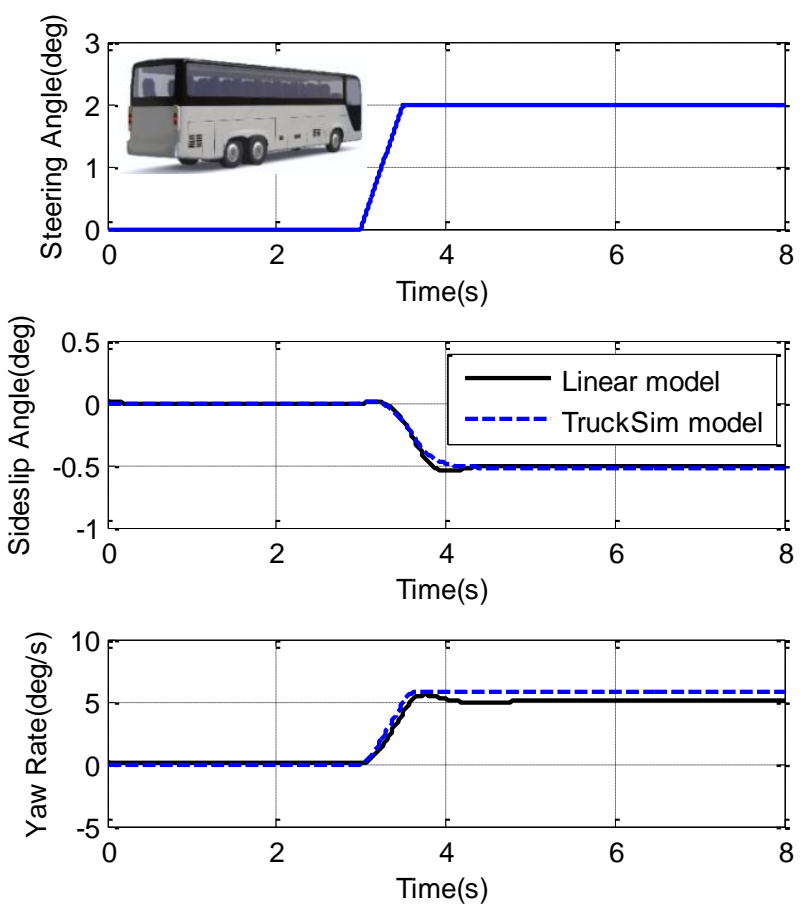

Fig. 2 Validation of the derived model by step steer

TABLE I PARAMETERS FOR A GENERIC THREE-AXLE VEHICLE

\begin{tabular}{lll} 
Parameter & Description(units) & Value \\
\hline$m$ & Vehicle mass $(\mathrm{kg})$ & 9415 \\
\hline$l_{f}$ & Distance of front axle from CG(m) & 3.5 \\
\hline$l_{r}$ & Distance of rear axle from CG(m) & 3.47 \\
\hline$l_{c}$ & Distance of central axle from CG(m) & 2.29 \\
\hline$l$ & Distance of front axle from rear axle(m) & 6.97 \\
\hline$l_{r c}$ & Distance of central axle from rear axle(m) & 1.22 \\
\hline$I_{z}$ & Yaw moment of inertia(kgm2) & 34685 \\
\hline$C_{f}$ & Total front wheels stiffness(N/rad) & $3.35 \mathrm{e} 5$ \\
\hline$C_{c}$ & Total central Wheels stiffness (N/rad) & $2.75 \mathrm{e} 5$ \\
\hline$C_{r}$ & Total rear wheels stiffness (N/rad) & $2.45 \mathrm{e} 5$ \\
\hline
\end{tabular}




\section{PARAMETER IDENTIFICATION AND CONTROL DESIGN}

\section{A. Parameter Identification}

The parameter identification (PI) of the unknown parameters is performed using an LS estimator. The estimator uses the measurements of steering angle $\delta$, extra yaw moment $M_{e}$, and yaw rate $r$, assuming available. In reality, these parameters are available for vehicles with advanced vehicle sensing technologies such as vehicles with GPS/IMU. Although these advanced sensing systems are not widely available for commercial cars yet, they could be equipped on other specific applications such as autonomous and military surveillance vehicles [12-13]. As for the vehicle side slip angle, it is usually estimated by other measurements such as the lateral acceleration and longitudinal speed of the vehicle from (1). In this research, we assume the side slip angle is available for measurement. Detailed explanations of the following basic procedures can be found in [14].

\section{1) Parametric Model}

The model (7) is in the form,

$$
\left[\begin{array}{c}
\dot{\beta} \\
\dot{r}
\end{array}\right]=\left[\begin{array}{ll}
a_{11} & a_{12} \\
a_{21} & a_{22}
\end{array}\right]\left[\begin{array}{l}
\beta \\
r
\end{array}\right]+\left[\begin{array}{cc}
b_{11} & 0 \\
b_{21} & b_{22}
\end{array}\right]\left[\begin{array}{c}
\delta \\
M_{e}
\end{array}\right]
$$

where $b_{11}=k_{11} a_{11}, k_{11}=-\frac{C_{f}}{C_{f}+C_{c}+C_{r}}, l_{c}=l-l_{f}-l_{r c}$,

$$
l_{r}=l-l_{f},
$$

$$
\begin{aligned}
& a_{21}=-\frac{l_{f} C_{f}-l_{c} C_{c}-l_{r} C_{r}}{I_{z}} \\
& =-\frac{l_{f}\left(C_{f}+C_{c}+C_{r}\right)}{I_{z}}+\frac{\left(C_{c}+C_{r}\right) l-C_{c} l_{r c}}{I_{z}} \\
& =k_{21} b_{21}+k_{22} b_{22}
\end{aligned}
$$

where $k_{21}=-\frac{C_{f}+C_{c}+C_{r}}{C_{f}}, k_{22}=\left(C_{c}+C_{r}\right) l-C_{c} l_{r c}$.

Separating the known terms from the unknown ones and applying the Laplace transform to $\beta$ and $r$ yields (10) and (11), respectively,

$$
\begin{gathered}
s \beta=a_{11} \beta+a_{12} r+k_{11} a_{11} \delta=a_{11}\left(\beta+k_{11} \delta\right)+a_{12} r \\
s r=a_{22} r+b_{21}\left(\delta+k_{21} \beta\right)+b_{22}\left(M_{e}+k_{22} \beta\right)
\end{gathered}
$$

Filtering both sides of the equation with the filter $\frac{1}{\Lambda(s)}=\frac{1}{s+l}$ ,we can express the system in the form of the static parametric model (SPM) as

$$
\begin{aligned}
& z_{1}=\theta_{1}^{* T} \phi_{1} \\
& z_{2}=\theta_{2}^{* T} \phi_{2}
\end{aligned}
$$

where $z_{1}=\frac{s}{\Lambda(s)} \beta, \quad \theta_{1}^{*}=\left[\begin{array}{ll}a_{11} & a_{12}\end{array}\right]^{T}$,

$$
\begin{aligned}
& \phi_{1}=\left[\frac{1}{\Lambda(s)} \beta+\frac{k_{11}}{\Lambda(s)} \delta \frac{1}{\Lambda(s)} r\right]^{T} \\
& z_{2}=\frac{s}{\Lambda(s)} r, \quad \theta_{2}^{*}=\left[\begin{array}{lll}
a_{22} & b_{21} & b_{22}
\end{array}\right]^{T} \text {, } \\
& \phi_{2}=\left[\frac{1}{\Lambda(s)} r \quad \frac{1}{\Lambda(s)} \delta+\frac{k_{21}}{\Lambda(s)} \beta \quad \frac{1}{\Lambda(s)} M_{e}+\frac{k_{22}}{\Lambda(s)} \beta\right]^{T} .
\end{aligned}
$$

In this case, $z_{i}$ and $\phi_{i}(i=1,2)$ are available for measurement or estimation since then can be generated by filtering the measurements $u$ and $x$.

\section{2) Estimation Model and Adaptive Law}

The estimation model has the same form as the SPM with the exception that the unknown parameter $\theta^{*}$ is replaced with its estimate at time $\mathrm{t}$, denoted by $\theta(t)$, i.e.,

$$
\hat{z}_{1}(t)=\theta_{1}^{T}(t) \phi_{1}(t)
$$

$$
\hat{z}_{2}(t)=\theta_{2}^{T}(t) \phi_{2}(t)
$$

and the estimation errors,

$$
\varepsilon_{i}(t)=\frac{z_{i}(t)-\hat{z}_{i}(t)}{m_{s i}^{2}},(i=1,2)
$$

where $m_{s i}$ is the normalizing signal designed to bound $\phi$ from above. A straightforward choice for $m_{s i}$ is $m_{s i}{ }^{2}=1+\alpha_{i} \phi_{i}^{T} \phi_{i}$ for any $\alpha_{i}>0$.

We use the recursive least-squares algorithm with forgetting factor to minimize the cost function,

$$
\begin{gathered}
\dot{\theta}_{i}(t)=P_{i}(t) \varepsilon_{i}(t) \phi_{i}(t), \theta_{i}(0)=\theta_{i 0} \\
\dot{P}_{i}(t)=\beta P_{i}(t)-P_{i}(t) \frac{\phi_{i}^{T} \phi_{i}}{m_{s i}{ }^{2}} P_{i}(t), P_{i}(0)=P_{i 0}=Q_{i 0}{ }^{-1},(i=1,2)
\end{gathered}
$$

\section{3) Stability and Convergence}

To guarantee the convergence of $\theta_{i}(t)$ to their actual values $\theta_{i}^{*}$, the input (for this application the steering angle) should include at least two different frequencies to ensure it is sufficiently rich signal and $\frac{\phi_{i}}{m_{s i}}$ is persistently exciting [14]. Although the scenario of varying vehicle mass and yaw inertia which affects the CG position of the vehicle is considered for the paper, the varying range of the two parameters are relatively fixed small range. Table 2 shows the range of the unknown parameters to be estimated. This is used as a parameters projection for a better estimation of convergence.

TABLE II RANGES OF UNKNOWN PARAMETERS

\begin{tabular}{llll}
\hline Parameters & Mass(kg) & Yaw inertia(kgm2) & lf(m) \\
\hline minimum & 9415 & 34685 & 3 \\
maximum & 11415 & 37486 & 4 \\
\hline
\end{tabular}




\section{B. Adaptive Reference Model}

The desired vehicle behavior is can be described by a reference model. The desired vehicle performance from the reference model is to have zero vehicle sideslip angle at the center of gravity in steady state and the desired yaw rate to be determined by the front steering angle and vehicle speed.

In steady state, variables can be solved in terms of the control input as

$$
\begin{gathered}
{\left[\begin{array}{c}
\beta_{s s} \\
r_{s s}
\end{array}\right]=-A^{-1}\left[\begin{array}{ll}
\frac{C_{f}}{m v_{x}} & \frac{l_{f} C_{f}}{I_{z}}
\end{array}\right]^{T} \delta} \\
r_{\mathrm{ss}}=G_{r} \delta
\end{gathered}
$$

where, $G_{r}=-\frac{-a_{21} b_{11}+a_{11} b_{21}}{a_{11} a_{22}-a_{21} a_{12}}$ is the steady-state gain of yaw rate, which leads to an under-steering behavior of the vehicle's stability. The reference model proposed is assumed to be a first-order system [15] as follows,

$$
r_{d}=W_{m}(s) \delta=\frac{G_{r}}{1+\tau s} \delta
$$

where $\tau$ is the desired time constant of yaw rate. This low-pass filter is used to filter out the noise in the yaw rate signal [16]. Applying the adaptive reference model from the parameters estimation results in $\hat{G}_{r}=-\frac{-\hat{a}_{21} \hat{b}_{11}+\hat{a}_{11} \hat{b}_{21}}{\hat{a}_{11} \hat{a}_{22}-\hat{a}_{21} \hat{a}_{12}}$.

\section{Controller Design}

The overall system including PI and control scheme is shown in Fig. 3. For yaw control, a model reference adaptive control (MRAC) is proposed. The desired vehicle behavior which is an LTI first order transfer function (15) is described by a reference model and is driven by a reference input. The control law is then developed so that the closed-loop plant has a transfer function equal to the reference model.

Consider the yaw rate of the plant,

$$
\dot{r}=a_{21} \beta+a_{22} \gamma+b_{21} \delta+b_{22} M_{e}
$$

where $a_{21}, a_{22}, b_{22}$ are constants but unknown. The control objective is to determine a bounded function $M_{e}=$ $f(t, \beta, r, \delta)$ such that the yaw rate is tracking the desired yaw rate from the reference model of (14), Rewriting the reference model yields

$$
(1+\tau s) r_{d}=G_{r} \delta \rightarrow \dot{r}_{d}=-\frac{1}{\tau} r_{d}+\frac{G_{r}}{\tau} \delta
$$

If the plant parameters are known, control law can be considered as

$$
M_{e}=k_{1} \beta+k_{2} \gamma+k_{3} \delta
$$

Substituting (17) into (15), results in the closed-loop system as

$$
\begin{aligned}
& \dot{r}_{c}=a_{21} \beta+a_{22} r_{c}+b_{21} \delta+b_{22}\left(k_{1} \beta+k_{2} r_{c}+k_{3} \delta\right) \\
& =\left(a_{21}+k_{1} b_{22}\right) \beta+\left(a_{22}+k_{2} b_{22}\right) r_{c}+\left(b_{21}+k_{3} b_{22}\right) \delta
\end{aligned}
$$

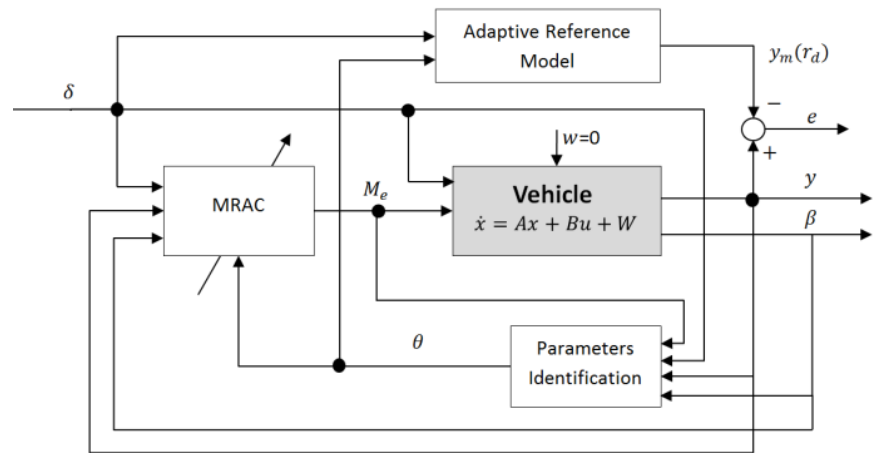

Fig.3 The PI and control scheme of whole system

The control objective is satisfied if controller parameters $k_{1}, k_{2}, k_{3}$ are carefully chosen, in a way that the closed-loop poles are stable and $\frac{\gamma_{c}}{\delta}=\frac{\gamma_{d}}{\delta}$, as follows:

$$
k_{1}=-\frac{a_{21}}{b_{22}}, k_{2}=-\frac{a_{21}}{b_{22}}, k_{3}=-\frac{a_{21}}{b_{22}}
$$

Replacing the unknown plant parameters with their estimates, the adaptive control law is established as follows:

$$
\begin{aligned}
& \hat{G}_{r}=-\frac{-\hat{a}_{21} \hat{b}_{11}+\hat{a}_{11} \hat{b}_{21}}{\hat{a}_{11} \hat{a}_{22}-\hat{a}_{21} \hat{a}_{12}}, \\
& \hat{k}_{1}=-\frac{\hat{a}_{21}}{\hat{b}_{22}}, \hat{k}_{2}=-\frac{1}{\tau \hat{b}_{22}}-\frac{\hat{a}_{22}}{\hat{b}_{22}}, \hat{k}_{3}=\frac{\hat{G}_{r}}{\tau \hat{b}_{22}}-\frac{\hat{b}_{21}}{\hat{b}_{22}} .
\end{aligned}
$$

\section{Simulations}

To verify the proposed MRAC, simulations are performed in MATLAB and Simulink. The simulation results include vehicle responses with different parameters sets (Fig. 4), parameters identification (Fig. 5). The behavior of yaw rate, and side slip angle using adaptive yaw control is also presented (Fig. 6).

\section{A. Different Parameter Sets}

To demonstrate the influence of varying parameters, different sets of mass, yaw inertia and CG position (lf) are simulated and the vehicle dynamics response and reference model are observed. Table 3 presents two possible parameters sets and were applied to the same bus. Fig. 4 shows a significant offset for both vehicle sideslip angle and yaw rate responses due to parameters changes. For the reference of yaw rate, the similar error happened between Para. Set 1 (reference 1) and Para. Set 2. This implies that the reference model will continuously change the values for reference output resulting in the controller to track inaccurate reference. However, an adaptive reference model is an on-line estimation and will adapt to the change of vehicle parameters.

\section{TABLE III TWO PARAMETERS SETS}

\begin{tabular}{llll}
\hline Para. Set & Mass(kg) & Yaw inertia(kgm2) & lf(m) \\
\hline 1 & 9415 & 34685 & 3.5 \\
2 & 10945 & 36185 & 3 \\
\hline
\end{tabular}



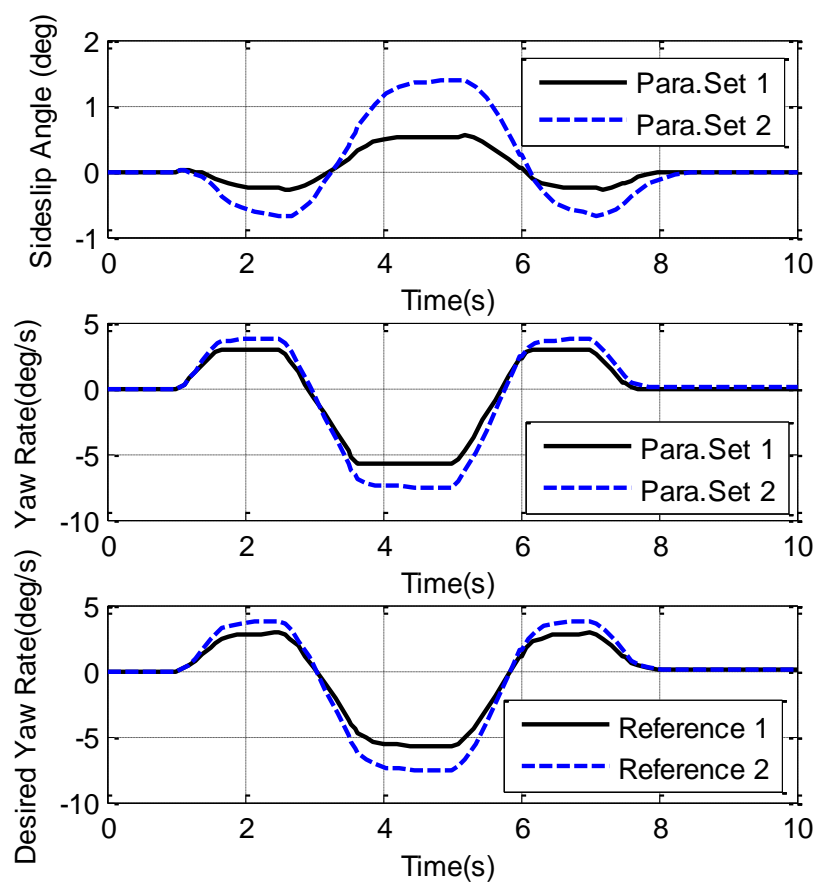

Fig.4 Vehicle responses with different parameters sets

\section{B. Results of PI and Adaptive Control}

Persistently exciting signal is desired to ensure that the unknown parameters converge to the actual values using the adaptive scheme. In practical driving condition, the driver's steering input is not a simple step input or sinusoidal input, but a combination of complex shaped inputs. For the sake of simulation, we apply the front wheel a slight steering angle incas follows,

$$
\delta=0.05+0.05 \sin 0.5 t+0.1 \sin t(\mathrm{deg})
$$

The unknown parameters were estimated using the steering input (20), which contains two different frequencies. Estimation results show that all parameters converge to their actual values within or around 35 seconds. Hence, the accuracy of the reference model is guaranteed. In Fig. 6, the first 10 seconds of the actual yaw rate behavior shows large fluctuations. This is due to the large fluctuations from parameters estimation module. They could be constrained within reasonable bounds in real application. However, after the 10th second, the actual yaw rate tracks the desired yaw rate precisely. From $36^{\text {th }}$ to $43^{\text {rd }}$ second, a steering input of double lane change is applied to the vehicle integrating with (20). Results show the actual yaw rate tracks the reference as well by generating an active yaw moment as the control action.

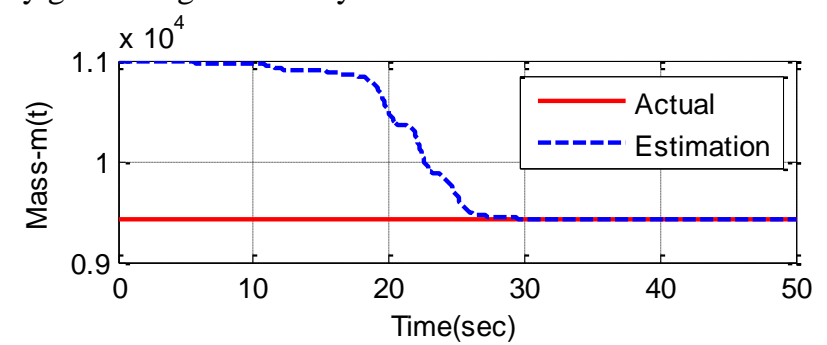

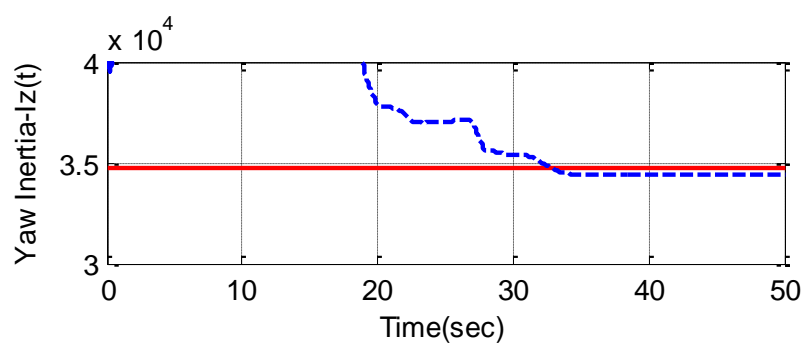

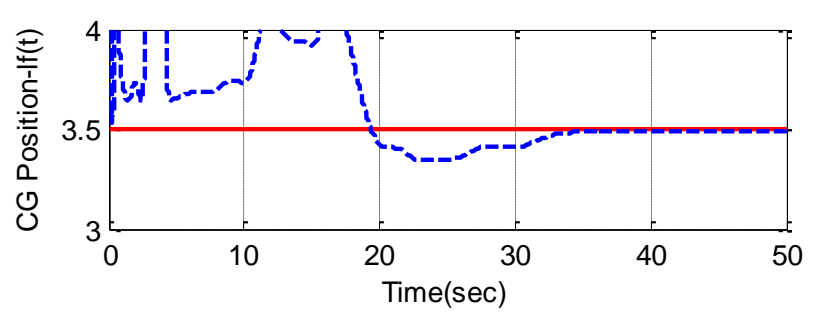

Fig.5 Parameters identification
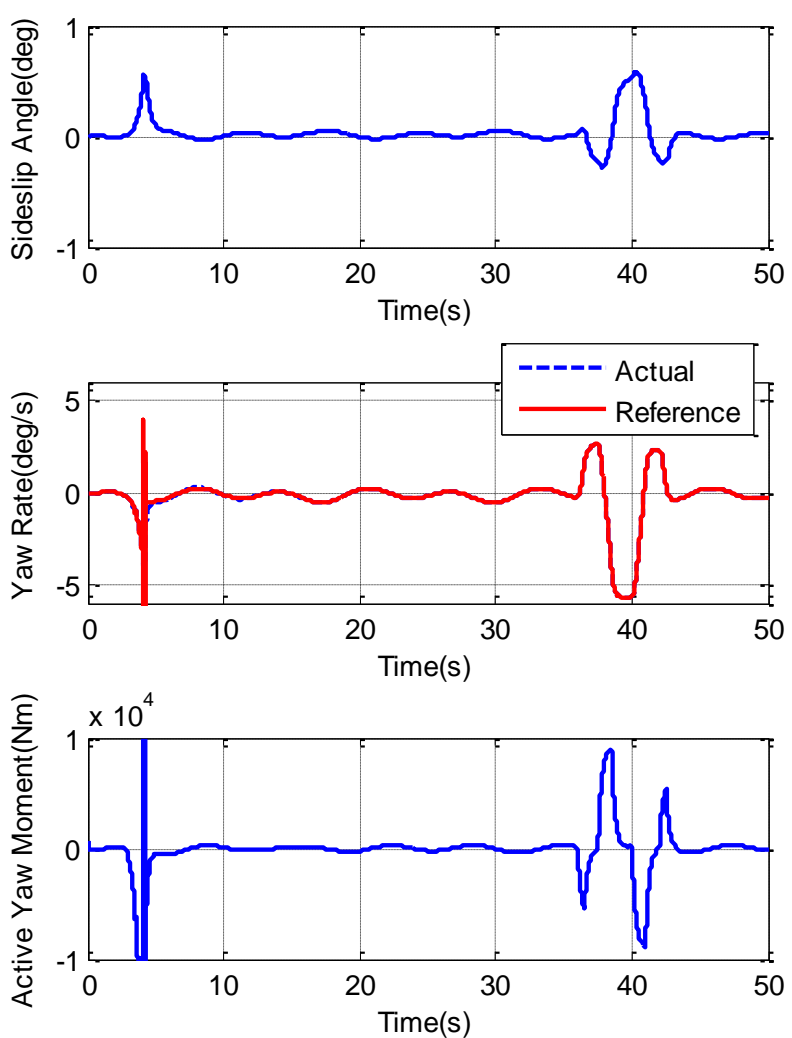

Fig.6 Adaptive yaw control

\section{CONCLUSIONS}

In this paper, the vehicle mass, yaw inertia and $\mathrm{CG}$ position identification and a MRAC with DYC were proposed, modeled and verified. By comparison, a reference model without adaptive parameters estimation is not accurate.

To estimate the unknown parameters and verify the MRAC, an interrelated system model was built and simulated with MATLAB and Simulink. The estimation results and time response of the yaw rate and vehicle sideslip show that the proposed method was effective in improving vehicle's yaw 
stability. For further study, a more comprehensive and realistic vehicle dynamic model is recommended and the plant should be tested in a real car or commercial software like TruckSim. Deeper merits of adaptive control strategy, along with robustness with parameter changes, i.e. tire nonlinear effects, will be also investigated and compared with the previous DYCs, i.e optimal control.

\section{REFERENCES}

[1] M. Shino and M. Nagai, "Yaw-moment control of electric vehicle for improving handling and stability," JSAE Review, vol. 22, no. 4, pp. 473480, 2001.

[2] A. Goodarzi, Avesta, N. Babak and E. Esmailzadeh, "Direct Yaw Moment Controller Design for Vehicle Dynamic Control Systems." Modelling, Simulation, and Optimization. pp. 120-124,2003.

[3] M. Canale, L. Fagiano, M. Milanese, and P. Borodani, "Robust vehicle yaw control using an active differential and IMC techniques," Control Engineering Practice, vol. 15, no. 8, pp. 923-941, 2007.

[4] B. Tabbache, A. Kheloui, and M. E. H. Benbouzid, "An Adaptive Electric Differential for Electric Vehicles Motion Stabilization," IEEE Transactions on Vehicular Technology, vol. 60, no. 1, pp. 104-110, 2011.

[5] J. Tjonnas and T. A. Johansen, "Stabilization of Automotive Vehicles Using Active Steering and Adaptive Brake Control Allocation," IEEE Transactions on Control Systems Technology, vol. 18, no. 3, pp. 545558, 2010.

[6] Y. Zhang, A. Khajepour, and B. Zhang, "Vehicle yaw dynamics control using Pulsed Active Rear Steering," IEEE International Conference on Mechatronics and Automation, pp. 263-268, 3-6 Aug.2014.
[7] M. Russo, R. Russo, and A. Volpe, "Car Parameters Identification by Handling Manoeuvres," Vehicle System Dynamics, vol. 34, no. 6, pp. 423-436, 2000.

[8] D. Wesemeier and R. Isermann, "Identification of vehicle parameters using stationary driving maneuvers," Control Engineering Practice, vol. 17, no. 12, pp. 1426-1431, 2009.

[9] S. Hong, C. Lee, F. Borrelli, and J. K. Hedrick, "A Novel Approach for Vehicle Inertial Parameter Identification Using a Dual Kalman Filter," IEEE Transactions on Intelligent Transportation Systems, vol. 16, no. 1, pp. 151-161, 2015.

[10] A. Soltani, A. Goodarzi, M. H. Shojaeefard, and A. Khajepour, "Vehicle dynamics control using an active third-axle system," Vehicle System Dynamics, vol. 52, no. 11, pp. 1541-1562, Aug. 2014.

[11] Y. Zhang, A. Khajepour, and Y. Huang, "Multi-axle/articulated bus dynamics modeling: a reconfigurable approach,"Vehicle System Dynamics, pp. 1-29, Sep. 2018.

[12] J. Wang, and M.F. Hsieh. "Vehicle yaw-inertia-and mass-independent adaptive steering control." Proceedings of the Institution of Mechanical Engineers, Part D: Journal of Automobile Engineering 223.9: pp.1101$1108,2009$.

[13] J. Wang, L. Alexander, and R. Rajamani, "Friction Estimation on Highway Vehicles Using Longitudinal Measurements," Journal of Dynamic Systems, Measurement, and Control, vol. 126, no. 2, p. 265, 2004.

[14] P. Ioannou and B. Fidan, "Adaptive Control Tutorial." Society for Industrial and Applied Mathematics, 2006.

[15] O. Mokhiamar and M. Abe, "Effects of model response on model following type of combined lateral force and yaw moment control performance for active vehicle handling safety," JSAE Review, vol. 23, no. 4, pp. 473-480, 2002.

[16] C. Fu, "Direct yaw moment control for electric vehicles with independent motors," PhD thesis, RMIT University, 2014. 